\title{
NIPBL: a new player in myeloid cell differentiation
}

Haematologica 2019

Volume 104(7):1332-1341

\section{Correspondence:}

ANNA PISTOCCHI

anna.pistocchi@unimi.it

Received: July 5, 2018.

Accepted: January 3, 2019.

Pre-published: January 10, 2019.

doi:10.3324/haematol.2018.200899

Check the online version for the most updated information on this article, online supplements, and information on authorship \& disclosures: www.haematologica.org/content/104/7/1332

(C)2019 Ferrata Storti Foundation

Material published in Haematologica is covered by copyright. All rights are reserved to the Ferrata Storti Foundation. Use of published material is allowed under the following terms and conditions:

https://creativecommons.org/licenses/by-nc/4.0/legalcode. Copies of published material are allowed for personal or internal use. Sharing published material for non-commercial purposes is subject to the following conditions:

https://creativecommons.org/licenses/by-nc/4.0/legalcode, sect. 3. Reproducing and sharing published material for commercial purposes is not allowed without permission in writing from the publisher.

\author{
Mara Mazzola, ${ }^{1 *}$ Gianluca Deflorian, ${ }^{2 *}$ Alex Pezzotta, ${ }^{1}$ Laura Ferrari, ${ }^{2}$ Grazia \\ Fazio, ${ }^{3}$ Erica Bresciani, ${ }^{4}$ Claudia Saitta,${ }^{3}$ Luca Ferrari, ${ }^{1}$ Monica Fumagalli, ${ }^{5}$ \\ Matteo Parma, ${ }^{5}$ Federica Marasca, ${ }^{6}$ Beatrice Bodega, ${ }^{6}$ Paola Riva, ${ }^{1}$ Franco \\ Cotelli, ${ }^{7}$ Andrea Biondi, ${ }^{3}$ Anna Marozzi, ${ }^{1}$ Gianni Cazzaniga ${ }^{3}$ and Anna Pistocchi ${ }^{1}$
}

${ }^{1}$ Dipartimento di Biotecnologie Mediche e Medicina Traslazionale, Università degli Studi di Milano, LITA, Segrate, Italy; '2 Istituto FIRC di Oncologia Molecolare, IFOM, Milano, Italy; ${ }^{3}$ Centro Ricerca Tettamanti, Clinica Pediatrica Università di Milano-Bicocca, Centro Maria Letizia Verga, Monza, Italy; ${ }^{4}$ Oncogenesis and Development Section, National Human Genome Research Institute, National Institutes of Health, Bethesda, MD, USA; ${ }^{5} \mathrm{Clinica}$ Ematologica e Centro Trapianti di Midollo Osseo, Ospedale San Gerardo, Università di Milano-Bicocca, Monza, Italy; ${ }^{~ I}$ stituto Nazionale di Genetica Molecolare "Romeo ed Enrica Invernizzi" (INGM), Milano, Italy and 'Dipartimento di Bioscienze, Università degli Studi di Milano, Milano, Italy.

* MM and GDF contributed equally to this work

\section{ABSTRACT}

The nucleophosmin 1 gene (NPM1) is the most frequently mutated gene in acute myeloid leukemia. Notably, NPM1 mutations are always accompanied by additional mutations such as those in cohesin genes RAD21, SMC1A, SMC3, and STAG2 but not in the cohesin regulator, nipped B-like (NIPBL). In this work, we analyzed a cohort of adult patients with acute myeloid leukemia and NPM1 mutation and observed a specific reduction in the expression of NIPBL but not in other cohesin genes. In our zebrafish model, overexpression of the mutated form of NPM1 also induced downregulation of nipblb, the zebrafish ortholog of human NIPBL. To investigate the hematopoietic phenotype and the interaction between mutated NPM1 and nipblb, we generated a zebrafish model with nipblb downregulation which showed an increased number of myeloid progenitors. This phenotype was due to hyper-activation of the canonical Wnt pathway: myeloid cells blocked in an undifferentiated state could be rescued when the Wnt pathway was inhibited by dkk1b mRNA injection or indomethacin administration. Our results reveal, for the first time, a role for NIPBL during zebrafish hematopoiesis and suggest that an interplay between NIPBL/NPM1 may regulate myeloid differentiation in zebrafish and humans through the canonical Wnt pathway and that dysregulation of these interactions may drive leukemic transformation.

\section{Introduction}

Acute myeloid leukemia (AML) is an aggressive hematologic malignancy of bone marrow characterized by the accumulation of immature myeloid blasts that show defective differentiation and function. ${ }^{1,2}$ Advances in cancer genomics have shown that relatively few recurrent somatic mutations give rise to human AML, with an average of five mutations in each case of de novo AML. ${ }^{3}$ These somatic mutations, which collectively determine the malignant phenotype, are serially acquired in clones of self-renewing hematopoietic stem cells (HSC), termed pre-leukemic HSC. ${ }^{4}$ The genes mutated in HSC that are relevant in the pathogenesis of AML have been divided into nine categories, including transcription-factor fusions, nucleophosmin (NPM1), tumor-suppressor genes, DNA-methylation-related genes, signaling genes, chromatin-modifying genes, myeloid transcription-factor genes, cohesin-complex genes and spliceosome-complex genes. ${ }^{5}$

NPM1, the most frequently mutated gene in $\mathrm{AML}$, is a phosphoprotein that normally resides in the nucleolus. ${ }^{6,7}$ More than 50 different reported mutations in human NPM1 result in aberrant cytoplasmic translocation of the protein, named 
$\mathrm{NPMc}+$, which functions as an oncogene in vitro ${ }^{8}$ and has a role in aberrant hematopoiesis in vivo. Indeed, murine models expressing NPMc+ in the hematopoietic lineage develop myeloproliferative disease ${ }^{9}$ and leukemia ${ }^{10,11}$ while the forced expression of human NPMc+ in zebrafish causes an increase in primitive early myeloid cells and definitive hematopoietic stem cells (HSC). ${ }^{12,13}$ It has also been demonstrated, with similar results in zebrafish and human AML blasts, that the expression of NPMc+ activates canonical Wnt signaling, providing insight into the molecular pathogenesis of AML bearing NPM1 mutations. ${ }^{12}$ Indeed, the canonical Wnt/ $\beta$-catenin pathway has been shown to be crucial for the regulation of HSC proliferation, differentiation and apoptosis. ${ }^{14}$

Recently, mutations in cohesin genes were found to be strongly correlated with NPM1 mutations although they do not seem to affect the prognosis of patients with AML. ${ }^{15}$ The cohesin complex is composed of different proteins that form a complex (SMC1, SMC3, RAD21, STAG1 and STAG2), and by additional regulator proteins (NIPBL, MAU2, ESCO1, ESCO2 and HDAC8). This multifunctional complex regulates the cohesion of sister chromatids during cell division, but also gene transcription and chromatin architecture. Recently, the genes of the cohesin complex were found to be mutated in almost $10 \%$ of patients with myeloid malignancies, while an additional $15 \%$ of patients had reduced expression of cohesin transcripts, suggesting a role for the cohesin complex in the pathogenesis of AML. ${ }^{5}$ In a cohort of patients studied by Thota and colleagues, ${ }^{15}$ the most frequently mutated genes of the cohesin complex were STAG2 (5.9\%), RAD21 (2\%), and SMC3 $(2 \%)$, whereas mutations in the other cohesins were less frequent $(<1 \%)$. Somatic mutations in cohesin subunits are mutually exclusive and, being mainly nonsense and frameshift mutations, result in a predicted lossof-function phenotype. ${ }^{16}$ It should be noted that cohesin mutations in AML, but not in other kind of tumors, ${ }^{17}$ are associated with a normal karyotype in malignant cells; therefore, the role of cohesins in tumor development is not correlated with their function in sister chromatid cohesion but rather with their role in mediating DNA accessibility to gene regulatory elements. ${ }^{15}$ Indeed, in vitro and in vivo models of cohesin haploinsufficiency show a delay in the differentiation of HSC, which are expanded in an immature state. ${ }^{18-21}$

In this work, we studied the expression of cohesin genes in a cohort of adults with AML and found a specific downregulation of NIPBL when NPM1 was mutated. Interestingly, we also found that nipblb was downregulated in our zebrafish model for NPMct expression. The zebrafish (Danio rerio) is a powerful model for studying hematologic diseases as it shares several hematopoietic genes with higher vertebrates, and mutations in human leukemia-causative genes disrupt normal hematopoiesis in zebrafish, suggesting a functional conservation of these genes during evolution. Our zebrafish model with loss-offunction of nipblb showed dysregulation of myeloid cell differentiation with increased numbers of myeloid precursors and a decrease of mature myeloid cells. The hematopoietic phenotype presented by nipblb-loss-of-function zebrafish embryos recapitulated the myeloid defects presented by embryos with NPMct overexpression and was due to hyper-activation of the canonical Wnt pathway. Indeed, overexpression of the $d k k 1 b$ Wnt inhibitor or indomethacin treatment rescued the phenotype.
Our study provides new insights into the molecular mechanisms underlying NIPBL function, identifying the canonical Wnt pathway as one of its targets and indicating that it plays a role with NPMc+ in the development of AML. Using the well-suited zebrafish model, we established a platform to further investigate the mechanisms through which NPMc+ and NIPBL might interact and contribute to leukemic transformation.

\section{Methods}

\section{Patients}

Diagnostic bone marrow samples from healty subjects and 40 adult patients affected by AML were collected and characterized as described in the Online Supplementary Methods. Patients' material was collected after obtaining informed consent (protocol ASGMA-052A approved on May 8 8 $^{\text {th }}, 2012$ by Azienda San Gerardo). The clinical features of the participants are reported in Online Supplementary Table S1. Human material and derived data were used in accordance with the Declaration of Helsinki.

\section{Animals}

Zebrafish embryos were raised and maintained according to international (European Union Directive 2010/63/EU) and national (Italian decree n. 26 of March $4^{\text {th }}$, 2014) guidelines on the protection of animals used for scientific purposes, as described in the Online Supplementary Methods.

\section{Reverse transcription and real-time quantitative polymerase chain reaction assays}

RNA was extracted from human and zebrafish embryos using TRIZOL reagents (Life Technologies, Carlsbad, CA, USA), following the manufacturer's protocol. Quantitative reverse transcriptase polymerase chain reaction (RT-PCR) experiments on human samples were performed with the Universal Probe Library system (Roche Diagnostics, Basel, Swiss), as described in the Online Supplementary Methods. Probes and primers are reported in Online Supplementary Tables S2 and S3.

\section{Western blotting}

Protein extracts were prepared, loaded and quantified as described in the Online Supplementary Methods. The antibodies used are listed in Online Supplementary Table S4. Images were acquired using an Alliance MINI HD9 AUTO Western Blot Imaging System (UVItec Limited, Cambridge, UK) and analyzed with the related software.

\section{In situ hybridization and immunofluorescent analyses}

Whole mount in situ hybridization (WISH) experiments, were carried out as described by Thisse et al. ${ }^{22}$ and in the Online Supplementary Methods. Pictures were acquired with a Leica DFC480 photo camera (Leica, Wetzlar, Germany). Immunostaining was performed as described previously. ${ }^{23}$ The antibodies used are listed in Online Supplementary Table S4. Images were acquired as described in the Online Supplementary Methods.

\section{Sudan black staining}

Sudan black staining was performed as described by Cvejic et al. ${ }^{24}$ and in the Online Supplementary Methods.

\section{Fluorescence-activated cell sorting analyses}

Embryo dissociation was performed as described previously. Fluorescence-activated cell sorting (FACS) analyses were performed as described in the Online Supplementary Methods on 
$\mathrm{Tg}$ (CD41:GFP) zebrafish embryos at 3 days post-fertilization (dpf) or PU.1-stained cells derived from embryos at $3 \mathrm{dpf}$.

\section{Injections and indomethacin treatment}

Injections were carried out on one- to two-cell stage embryos. Morpholinos were injected as described in the Online Supplementary Methods. Human NPM1 and NPMc+ and zebrafish $d k k 1 b$ mRNA were generated as previously described ${ }^{12}$ and injected as detailed in the Online Supplementary Methods. Indomethacin (Sigma-Aldrich St. Louis, MO, USA) treatment was administered as described by North et al. ${ }^{26}$

\section{Confocal imaging}

Previously immunostained $3 \mathrm{dpf}$ embryos were equilibrated and mounted in $85 \%$ glycerol solution in phosphate-buffered saline and imaged using a TCS-SP2 confocal microscope (Leica), with 40X oil immersion objective, $488 \mathrm{~nm}$ argon ion and $405 \mathrm{~nm}$ diode lasers. For each sample, single stack images were acquired.

\section{Statistical analyses}

For quantitative RT-PCR experiments, data were statistically analyzed applying one-way analysis of variance (ANOVA) or $t$ tests, defining $P \leq 0.05\left(^{*}\right), P \leq 0.01\left(^{* *}\right)$, and $P \leq 0.001\left(^{* * *}\right)$ as statistically significant values. ${ }^{27}$ Data were analyzed using the comparative $\Delta \Delta \mathrm{Ct}$ method. Both t- test and standard deviation (SD) values refer to data from triplicate samples. In zebrafish at least three different experiments were done for each analysis. For cell counts and phenotypic analyses, statistical analysis was performed by the $\chi^{2}$ test, with Yates correction when needed.

\section{Results}

Cohesin expression in subgroups of adult patients with acute myeloid leukemia carrying NPM1 mutations and in a zebrafish model of NPMc+ expression

It was recently reported that somatic mutations in cohesin genes are frequently associated with NPM1 mutations ${ }^{28}$ but not with other common AML mutations; however, the expression levels of cohesins have never been analyzed. We, therefore, decided to investigate the presence of alterations in the expression of cohesin genes in a cohort of 40 adult AML patients stratified into two homogeneous molecular subgroups according to the absence or presence of a NPM1 mutation (NPMc+). Each subgroup was analyzed by means of quantitative RT-PCR for the expression of the cohesin genes SMC1A, SMC3, NIPBL and RAD21 (Figure 1A-D). Interestingly, only NIPBL showed a significantly decreased expression in AML patients carrying a NPM1 mutation (Figure 1B). Conversely, when the same patients were divided into subgroups depending on the presence or absence of a FLT3-internal tandem duplication (ITD), none of the cohesin genes analyzed was differently expressed between the two subgroups (Online Supplementary Figure S1).

We also used quantitative RT-PCR to analyze the expression of cohesin genes in a previously generated and well-characterized zebrafish model with human NPMc+ overexpression. ${ }^{12,13}$ The injection of 100 pg/embryo of $N P M c+$ transcript led to a significant downregulation of nipblb in the whole embryo at $3 \mathrm{dpf}$, while the expression of other cohesin genes analyzed was unaltered or even increased (Figure 1E). We performed a stage-dependent analysis to determine at which developmental stage the overexpression of NPMc+ started to downregulate nipblb expression in the whole embryo. We observed a significant decrease of nipblb expression starting from 48 hours post-fertilization (hpf), while no differential decreases between controls and NPMc+ mRNA-injected embryos were observed at 24 and $36 \mathrm{hpf}$ (Figure 1F). To further verify that the downregulation of zebrafish nipblb was strictly dependent on the forced expression of NPMc+, we injected different doses of the NPMc+ transcript (25 pg/embryo, $75 \mathrm{pg} / \mathrm{embryo}$ and $125 \mathrm{pg} / \mathrm{embryo}$ ) and found a significant inverse correlation between reduced nipblb expression and increased NPMc+ (Figure 1G). Moreover, western blot studies of embryos at $3 \mathrm{dpf}$ confirmed that the injection of NPMc+ mRNA, at a dose of $100 \mathrm{pg} /$ embryo, caused a reduction of the level of Nipbl protein in comparison to the level in control-injected embryos (Figure 1H).

\section{nipblb loss-of-function and NPMc+ overexpression generate hematopoietic defects in zebrafish embryos}

Since NIPBL is downregulated in AML patients in association with NPM1 mutations, we wondered whether nipblb knockdown might generate defects in myeloid cell differentiation. To investigate this hypothesis, we downregulated nipblb in zebrafish by injecting a nipblb antisense morpholino targeting the ATG region (nipblb-MO), as previously described for the knockdown of the Nipbl protein. ${ }^{29,30}$ In AML patients, HSC and immature myeloid blasts are increased. Therefore, to screen for myeloid defects in conditions resembling those of AML patients, we analyzed the same cell populations in nipblb-MO injected embryos. Knockdown of nipblb did not lead to significant differences in the HSC, as shown by confocal images of the caudal hematopoietic tissues (CHT) of $\mathrm{Tg}\left(\mathrm{CD} 41: G F P\right.$ ) embryos ${ }^{31}$ at $3 \mathrm{dpf}$ (Figure 2A,B) and by cytofluorimetric analyses to quantify the CD41:GFPlow HSC cells in comparison to those in controls (Figure 2D,E,G). On the other hand, at the same stage, nipblb-MO injected embryos showed an increase of myeloid progenitors, positive for the PU.1 antibody (Figure 2H,I; FACS in $\mathrm{K}, \mathrm{L}, \mathrm{N}$ ). These observations were also confirmed by means of WISH techniques with the HSC marker cmyb and the myeloid progenitor marker spitb with relative quantifications of the observed phenotypes, and RT-qPCR analyses (Online Supplementary Figure S2). Moreover, to verify whether the increased myeloid cells were blocked in an undifferentiated state, we used Sudan black staining to visualize mature myeloid cells. ${ }^{24}$ At 4 dpf we observed a reduction of neutrophils (Figure 2O,P).

It was previously reported that the forced expression of human NPMc+ in zebrafish embryos increased the HSC population at 30-36 $\mathrm{hpf}^{13}$ To further analyze whether the hematopoietic defects are still present later during hematopoiesis and are extended to myeloid cells, we injected the embryos with NPMc+ mRNA (100 pg/embryo) and analyzed the hematopoietic phenotype. The CD41:GFPlow cells in the CHT of NPMc+-injected embryos were expanded in comparison to those in the controls (Figure 2A,C; FACS in D,F,G), as were PU.1-positive myeloid precursors (Figure 2H,J; FACS in $\mathrm{K}, \mathrm{M}, \mathrm{N}$ ). Sudan black staining showed a decrease in mature myeloid cells in NPMct-injected embryos in comparison to those in controls (Figure 2O,Q). Moreover, the expression levels of cmyb and spitb, investigated by WISH and quantitative RT-PCR techniques, corroborated our previous findings (Online Supplementary Figure S3).

To confirm the specificity of the hematopoietic defects 

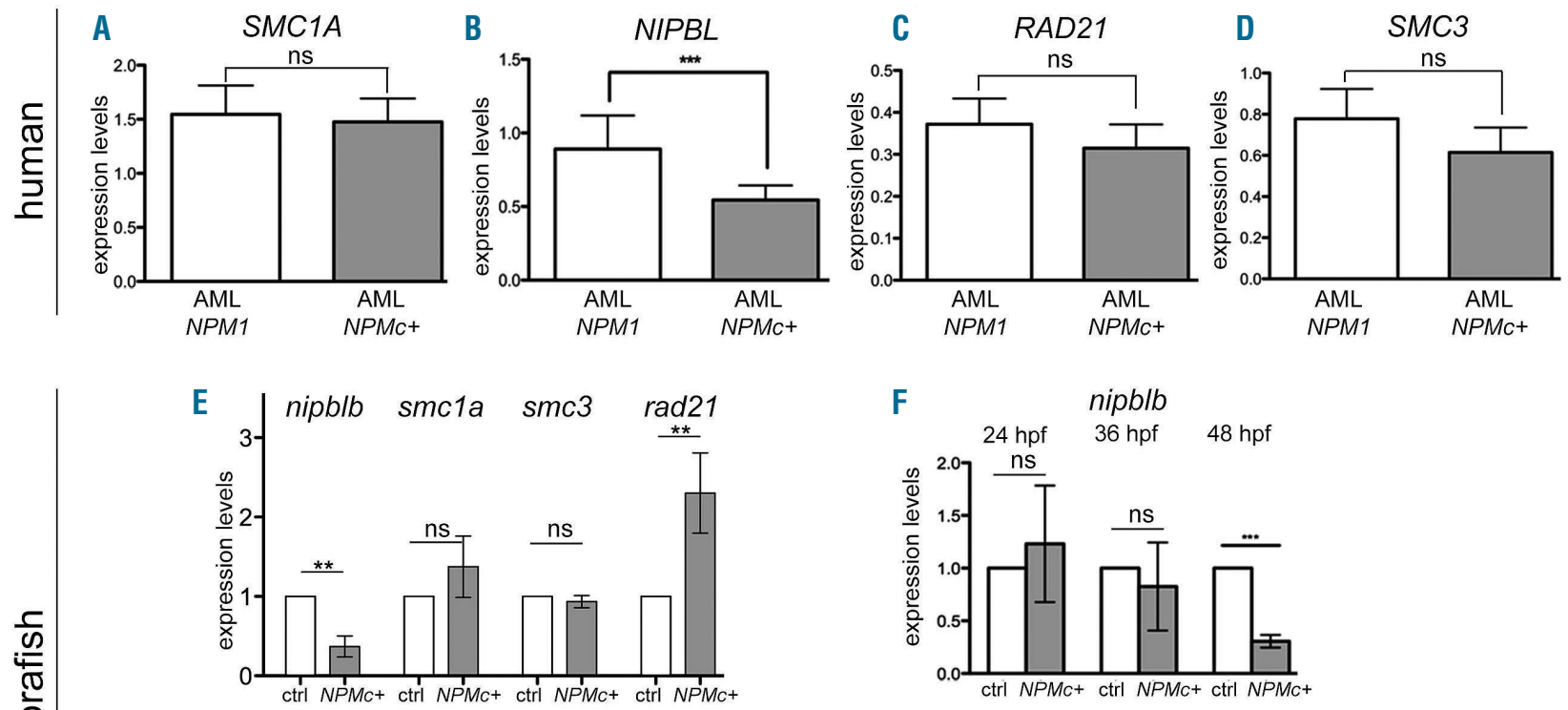

G

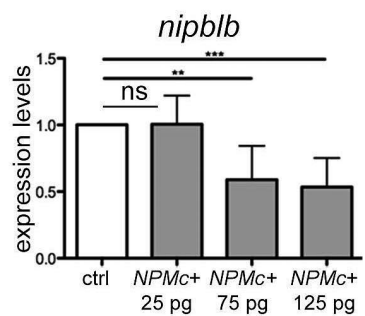

H

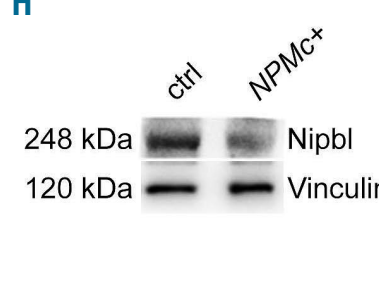

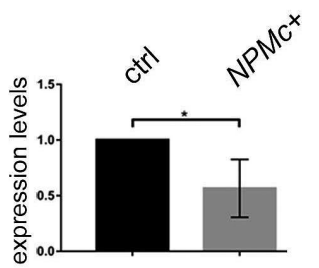

Figure 1. Expression of cohesin genes in bone marrow samples from adults with acute myeloid leukemia divided into two subgroups according to the absence or presence of NPM1 mutations and in a zebrafish model for NPM1 mutation (NPMc+). (A-D) Quantitative reverse transcriptase polymerase chain reaction (RT-qPCR) analyses in 40 adults with acute myeloid leukemia indicated that the expression of NIPBL was decreased when NPM1 was mutated. The expression levels of the other cohesin genes analyzed did not correlate with the presence of NPM1 mutations. (E) RT-qPCR analyses of cohesin gene expression in a zebrafish model with overexpression of human NPMc+ showed that the expression of nipblb was downregulated in comparison to that of controls at 3 days post-fertilization (dpf). (F) RTqPCR time course analyses at 24,36 and 48 hours post-fertilization (hpf) of nipblb expression in embryos injected with NPMc+ transcript. nipblb was significantly downregulated at $48 \mathrm{hpf}$. (G) RT-qPCR analyses of nipblb expression in zebrafish embryos at 3 dpf injected with different doses of human NPMc+. The downregulation of nipblb was significantly dependent on the dose of NPMc+ injected. $(\mathrm{H})$ Western blot analyses of Nipbl protein expression in embryos at $3 \mathrm{dpf}$ injected with $100 \mathrm{pg} /$ embryo of NPMc+ transcript. Vinculin marker was used for normalization. Western blot images were processed as described in the Online Supplementary Methods. $* P<0.05, * * P<0.01$ and $* * * P<0.001$. ns: non-significant; AML: acute myeloid leukemia; MO: morpholino; ctrl: control.

obtained with nipblb-MO injection, we used a second morpholino targeting the 5'UTR region of nipblb (5'UTRnipblb-MO), which has been previously used and validated. ${ }^{29}$ The injection of this second morpholino reduced the Nipbl protein levels, as determined by western blot analyses, confirming its efficacy in blocking protein production. It also recapitulated the hematopoietic defects observed with the injection of the ATG morpholino, showing an increased number of myeloid precursors positive for spitb and a decreased number of mature myeloid cells. Moreover, when co-injected at subcritical doses, the two nipblb-MO cooperated to induce the myeloid phenotype (Online Supplementary Figure S4).

\section{nipbl downregulation and NPMc+ overexpression both induce the hyper activation of the canonical Wnt pathway}

Previous experimental and clinical evidence suggested a close correlation between AML development and canonical Wnt pathway dysregulation, especially in patients with NPM1 mutations..$^{12}$ To examine whether nipblb and $N P M c+$ regulate the activation status of the canonical Wnt pathway, we used the $\operatorname{Tg}(T O P d G F P)$ transgenic line that bears the GFP reporter gene under the control of four enhancers and the basal promoter of lef1, a $\beta$-catenindependent transcription factor. ${ }^{32} \mathrm{Tg}(T O P d G F P)$ embryos were injected with nipblb-MO or NPMc+ mRNA and the expression of gfp and axin2, one of the direct targets of activated $\beta$-catenin, were analyzed by quantitative RTPCR at 3 dpf. ${ }^{33}$ Both genes were upregulated following nipblb knockdown or NPMc+ overexpression, indicating a hyper-activation of the canonical Wnt pathway (Figure $3 \mathrm{~A}, \mathrm{~B})$. To determine whether the Wnt signaling was enhanced specifically in hematopoietic cells, confocal images of the CHT of $T g(T O P d G F P)$ embryos were analyzed (Figure 3C). GFP' cells were increased in both nipblb$\mathrm{MO}$ - and NPMct-injected embryos (10 increased/10 scored). Specifically, nipblb downregulation almost doubled the number of $\mathrm{GFP}^{+}$cells in the CHT $(n=27 \pm 5)$ in comparison to the number in controls $(n=14 \pm 3$ ), (Figure $3 \mathrm{D}, \mathrm{E}$, quantification in I). NPMc+ overexpression also increased the number of GFP+ cells in the CHT $(n=20 \pm 4)$ in comparison to the number in controls $(n=14 \pm 3$ ), (Figure $3 \mathrm{D}-\mathrm{G}$, quantification in I). To further demonstrate that the increase of $\mathrm{GFP}^{+}$cells in the CHT was due to the hyperactivation of the canonical Wnt pathway, we injected the 
ctrl
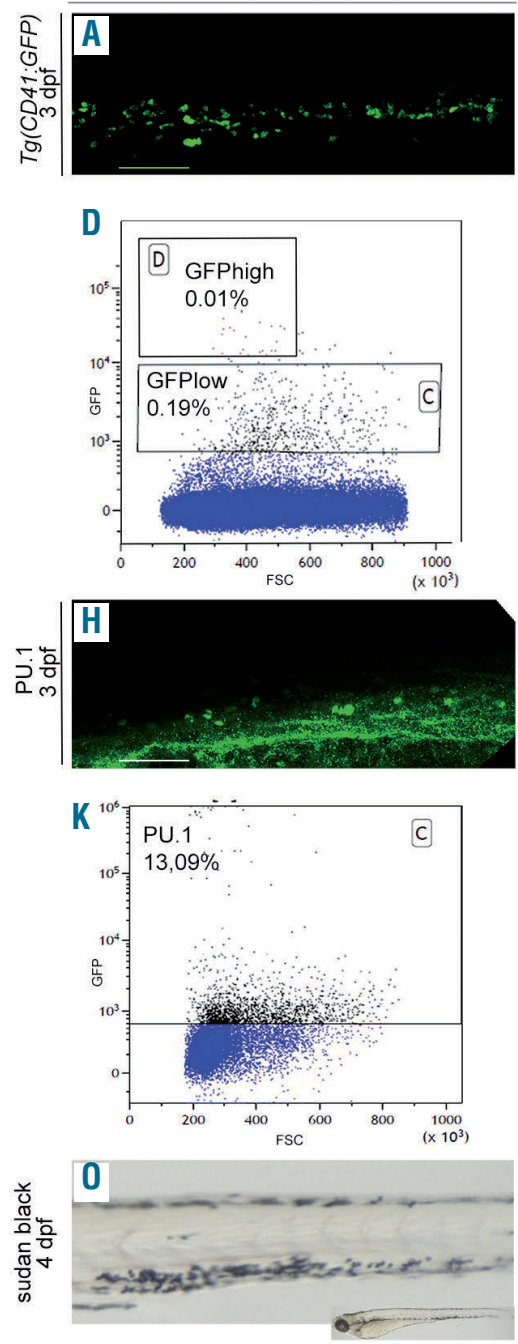

nipblb-MO

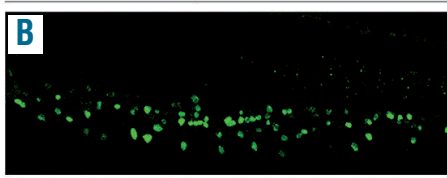

E
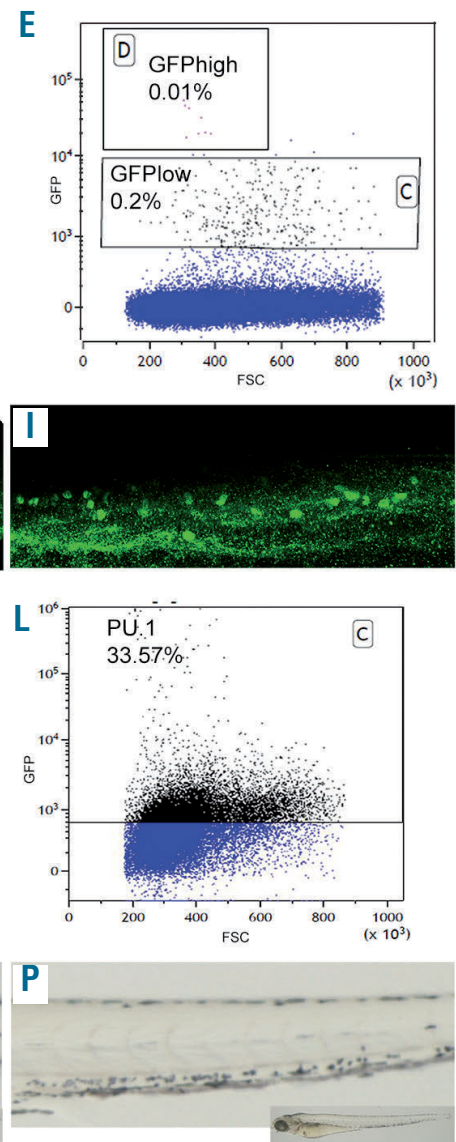

NPMc+
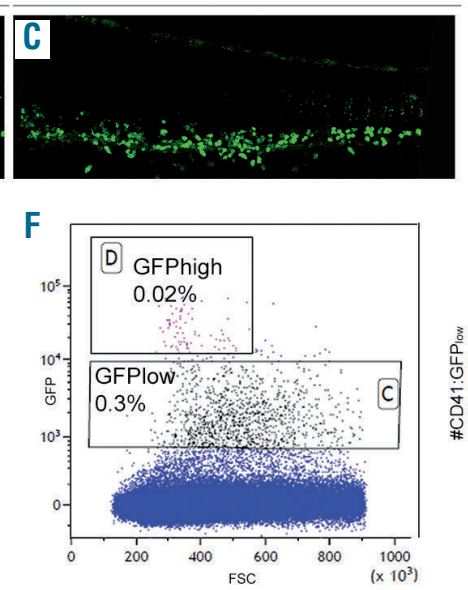

G
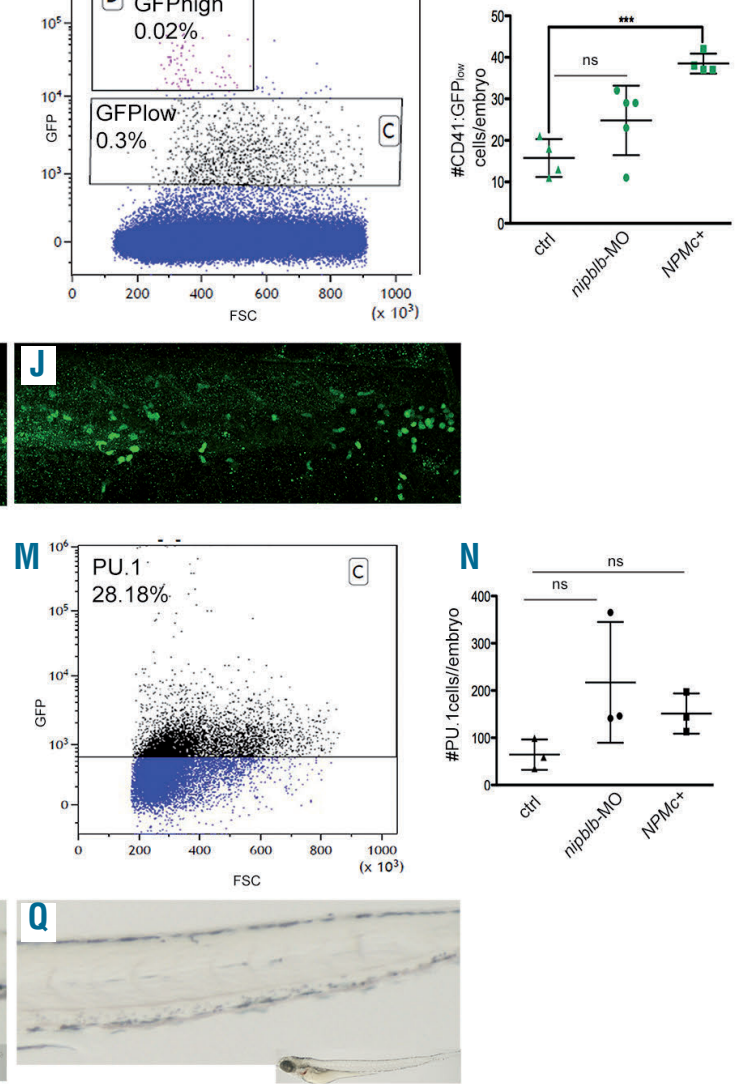

Figure 2. Myeloid cell differentiation is affected in nipblb-MO and NPMc+ mRNA injected embryos. (A-C) Confocal analyses of CD41:GFP hematopoietic stem cells (HSC) from controls, nipblb-MO and NPMc+ mRNA injected embryos at 3 days post-fertilization (dpf). The number of green fluorescent protein (GFP)-positive HSC was slightly increased in nipblb-MO, but significantly increased in NPMc+ embryos in comparison to controls. (D-G) Fluorescence-activated cell sorting (FACS) analyses on GFPlow-positive HSC. (H-J) Confocal analyses of PU.1-positive myeloid precursor cells from controls, nipblb-MO and NPMc+ mRNA injected embryos at 3 dpf. The numbers of GFP-positive myeloid precursors cells were increased in both nipblb-MO and NPMc+ embryos in comparison to controls. (K-N) FACS analyses on Pu.1 GFP-positive cells. (0-Q) Sudan black staining for mature myeloid cells in controls, nipblb-MO and NPMc+ mRNA injected embryos at 4 dpf. The mature myeloid cells were diminished in both nipblb-MO and NPMc+ embryos in comparison to controls. Images were processed as described in the Online Supplementary Methods. The scale bar represents $100 \mu \mathrm{m}$. $* * * P<0.001$. ns: non-significant; MO: morpholino; CHT: caudal hematopoietic tissue; ctrl: control.

Wnt inhibitor $d k k 1 b$ mRNA (50 pg/embryo). ${ }^{12}$ We validated the efficiency of $d k k 1 b$ mRNA injection as the $\mathrm{GFP}^{+}$ cells were diminished or absent in the hindbrain ventricle and in the CHT of the Tg(TOPdGFP) embryos in comparison to controls (Online Supplementary Figure S5). The coinjection of $d k k 1 b$ in nipblb-MO or NPMc+ mRNA-injected embryos rescued the number of $\mathrm{GFP}^{+}$cells in the CHT (10 rescued/10 scored for both) or even diminished the $\mathrm{GFP}^{+}$cells $(\mathrm{n}=8 \pm 3$ for nipblb-MO/dkk1b; $\mathrm{n}=10 \pm 3$ for $N P M c+/ d k k 1 b)$ (Figure $3 \mathrm{~F}-\mathrm{H}$, quantification in I). Moreover, using the Wnt reporter line Tg(TOPdGFP) we verified that, following nipblb-MO injection, the canonical Wnt pathway appeared downregulated at $24 \mathrm{hpf}$ but was then hyper-activated at $48 \mathrm{hpf}$ (Online Supplementary Figure
S6). This observation confirmed our previous work on a zebrafish model for nipblb-loss-of-function in which we observed downregulation of the canonical Wnt pathway at $24 \mathrm{hpf}$ and correlated this with the neurological defect presented by patients affected by Cornelia de Lange syndrome (CdLS). ${ }^{30}$

Having seen hyper-activation of the canonical Wnt pathway in our zebrafish model with nipblb downregulation, we also analyzed this in humans with AML by measuring the expression of AXIN2, previously used as a reporter of canonical Wnt pathway activation in AML patients. ${ }^{12}$ We did not observe a significant increase in AXIN2 expression in our cohort of AML patients with NIPBL downregulation $(N I P B L<1)$ in comparison to that in patients with normal or 
A

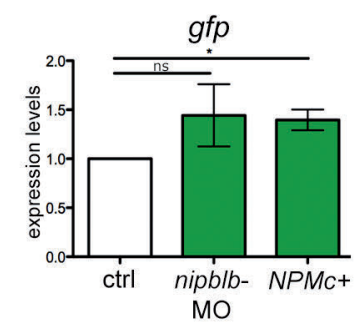

B

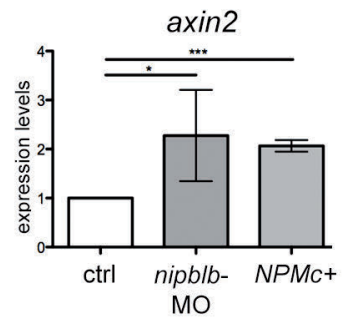

C

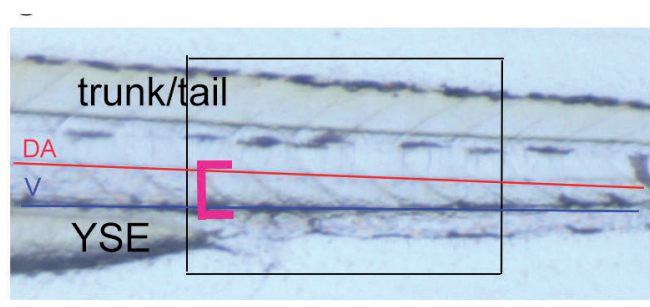

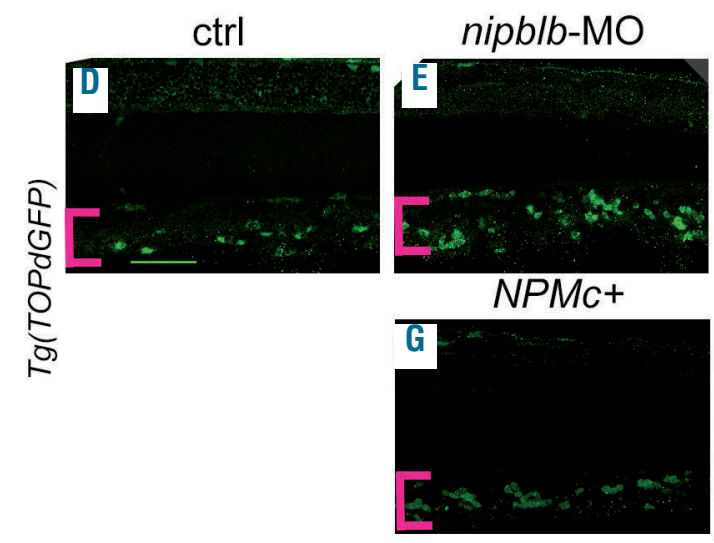

nipblb-MO/dkk1b

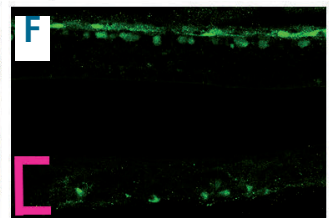

$N P M c+/ d k k 1 b$

$\mathrm{H}$

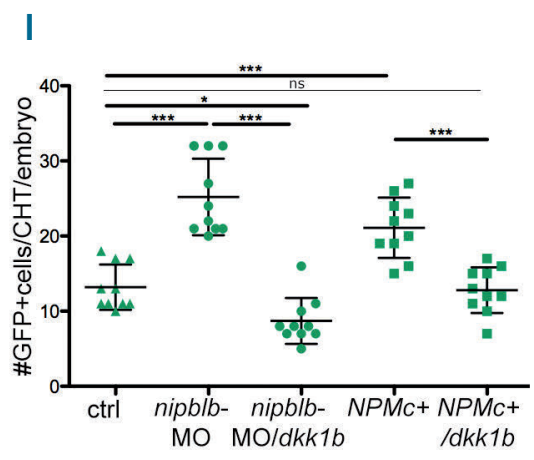

Figure 3. Canonical Wnt signaling is hyper activated in nipblb-MO and NPMc+ Tg(TOPdGFP) injected embryos at 3 days post-fertilization. (A, B) Quantitative reverse transcriptase polymerase chain reaction analyses of gfp and axin2 expression in nipblb-MO and NPMc+ Tg(TOPdGFP) injected embryos indicated an increase of canonical Wnt activation status in comparison to that in controls. (C) Scheme of the trunk-tail region of embryos. Confocal images were always taken of the same region of the embryo, comprising the tip of the yolk sack extension (YSE) between the dorsal aorta (DA, red line) and the vein (V, blue line) as indicated by pink brackets. (D-H) Confocal images of the caudal hematopoietic tissue (CHT) of Tg(TOPdGFP) embryos injected with nipblb-MO (E) and NPMc+ mRNA (G) showed an increase of $\mathrm{GFP}^{+}$cells in comparison to controls (D). Co-injection of the Wnt inhibitor dkk1b mRNA rescued the number of GFP ${ }^{+}$cells (F-H). (I) Quantification of GFP ${ }^{+}$cells in the selected region of the $\mathrm{CHT}$. Images were processed as described in the Online Supplementary Methods. The scale bar represents $100 \mu \mathrm{m}$. $* P<0.05, * * P<0.01$ and $* * * P<0.001$. ns: non-significant; MO: morpholino; GFP: green fluorescent protein; ctrl: control.

increased expression of NIPBL (NIPBL $>1)$ (Online Supplementary Figure S7). Further analyses in larger cohorts will be necessary to determine the correlation between NIPBL expression and the canonical Wnt pathway.

\section{Hyper-activation of canonical Wnt signaling in hematopoietic stem cells impairs myeloid differentiation}

To determine which type of hematopoietic cell in the CHT showed hyper-activation of the canonical Wnt pathway following nipblb-MO or NPMc+ mRNA injections, we sorted the CD 41:GFPlow cells $(0.8 \%$ control, $1 \%$ nipblb$\mathrm{MO}, 1.1 \% N P M c+)$ from $3 \mathrm{dpf}$ embryos injected with nipblb-MO or NPMc+ mRNA and observed a modest increase in axin2 expression in these cells in comparison to the expression in CD41:GFPlow cells from controls (Figure 4A$\left.A^{\prime \prime \prime}\right)$. Moreover, we performed immunofluorescence staining with the Active $\beta$-catenin (Active $\beta$-cat) antibody for the Wnt pathway and GFP antibody for HSC cells of the CD41:GFP embryos. The number of GFP/Active $\beta$-cat double-positive cells present in the CHT of 3-dpf embryos injected with nipblb-MO or NPMc+ mRNA (5 double-positive cells indicated by the arrows in the CHT of Figure $4 C^{\prime \prime}-\mathrm{D}$ ") was higher than that in controls (2 double-positive cells indicated by the arrows in the CHT of Figure $4 \mathrm{~B}$ "), demonstrating that the Wnt pathway was activated specifically in HSC (Figure 4B-D").

The myeloid differentiation defects presented by nipblb-MO and NPMc+ mRNA injected embryos at $3 \mathrm{dpf}$ were caused by hyper-activation of the canonical Wnt pathway. Inhibiting this pathway, either by $d k k 1 b$ mRNA injection or by treatment with the Wnt pharmacological inhibitor indomethacin, ${ }^{26}$ rescued the hematopoietic phenotype. Indeed, by WISH we showed that the increased number of myeloid precursors positive for spilb observed in nipblb-MO or NPMc+ mRNA injected embryos, returned to levels comparable to those in controls (Figure $5 A-G)$.

NPMc+ and nipblb cooperation in the hyper-activation of canonical Wnt signaling and myeloid defects

The evidence that we had collected so far showed that NPMc+ downregulates NIPBL, both in human AML patients and in zebrafish. We also demonstrated that dysregulation of both genes impairs myeloid differentiation through hyper-activation of the canonical Wnt pathway. To further address a possible cooperation between the two genes, we performed dose-response assays. We injected subcritical doses of nipblb-MO (0.6 pmol/embryo) or NPMc+ mRNA (50 pg/embryo), which singularly did not cause an increase in $\mathrm{GFP}^{+}$cells in the CHT of $T g$ (TOPdGFP) embryos (Figure 6B,C) in comparison to controls (Figure 6A, quantification in $\mathrm{E}$ ), or in spilb-positive myeloid progenitors (Figure $6 \mathrm{G}, \mathrm{H}$ ) in comparison to the number of cells in controls (Figure 6F, quantification in J). When co-injected, subcritical doses of nipblb$\mathrm{MO} / \mathrm{NPMc}+\mathrm{mRNA}$ recapitulated the phenotype previously observed following injections of full doses, with an 
A

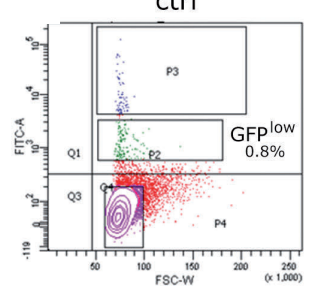

$A^{\prime}$

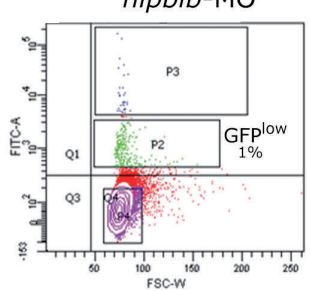

A"

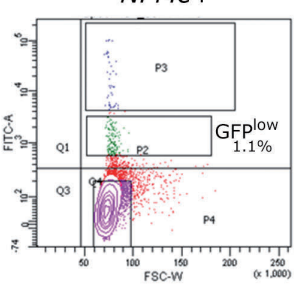

A"'

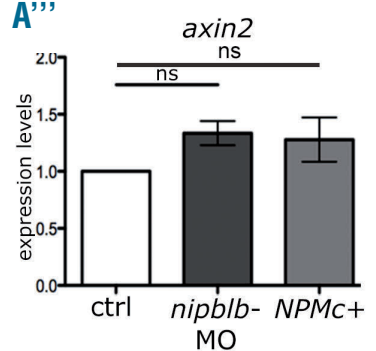

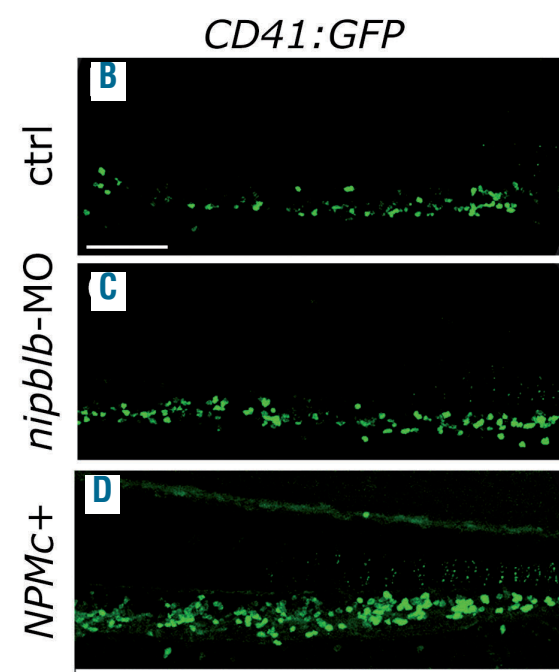

Active $\beta$ cat
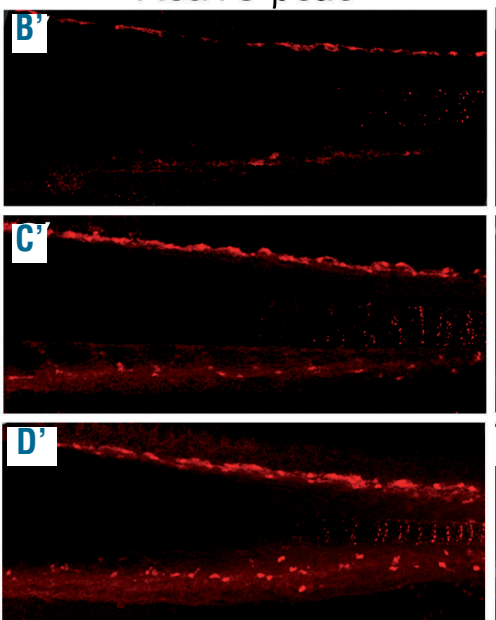
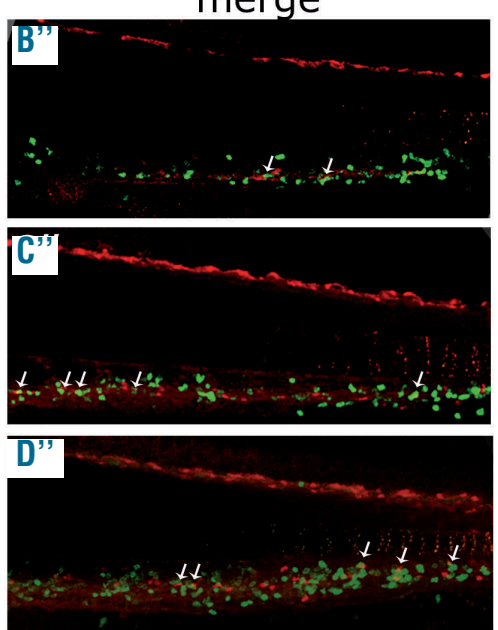

Figure 4. The canonical Wnt pathway is hyper-activated specifically in hematopoietic stem cells. (A-A") Fluorescence-activated cell sorting analysis of CD41:GFPlow cells from controls (A), nipblb-MO (A') and NPMc+ mRNA (A') injected embryos at 3 days post-fertilization (dpf) and quantitative reverse transcriptase polymerase chain reaction analysis of axin2 expression on sorted cells (A"'). (B-D") Immunofluorescence staining with green fluorescent protein (GFP) for hematopoietic stem cells. (B-D) and Active $\beta$-catenin for Wnt activation (Active $\beta$-cat) (B'-D') antibodies. Merging of the two signals (B'-D") showed an increased number of GFP/Active $\beta$ cat double-positive cells (arrows) at $3 \mathrm{dpf}$ in the caudal hematopoietic tissue of embryos injected with nipblb-MO or NPMc+ mRNA in comparison to the number in controls. Images were processed as described in the Online Supplementary Methods. The scale bar represents $100 \mu \mathrm{m}$. ${ }^{*} P<0.05$. ns: non-significant; ctrl: control; MO: morpholino.

increased number of $\mathrm{GFP}^{+}$cells in the CHT of the $T g(T O P d G F P)$ embryos and an enhanced expression of spilb (Figure 6F-I, quantification of the phenotypes in J), indicating a cooperation between NPMc+ and nipblb downregulation.

\section{Discussion}

In this work, we built on the observation that, in addition to the cohesin mutations detected in $10 \%$ of patients with myeloid malignancies, low expression of cohesin genes was present in an additional $15 \%$ of patients showing similar expression signatures as those with somatic cohesin mutations. ${ }^{28} \mathrm{We}$ therefore investigated the expression of cohesin genes in our cohort of 40 adult AML patients divided according to the absence/presence of NPM1 mutation. We chose to analyze NPM1 mutations as they are pivotal in AML but likely insufficient by themselves to cause malignant transformation, requiring the cooccurrence of other mutations such as FLT3-ITD or RAS. ${ }^{9,34}$ In addition, a correlation between NPM1 mutations and somatic mutations in cohesin genes has already been reported, ${ }^{28}$ although the specific loss-of-function of cohesins has never been investigated in association with NPM1 mutations. Among the cohesin genes analyzed in our cohort of adult AML patients, we found that only
NIPBL showed a lower expression when NPM1 was mutated. NIPBL is a regulator of the cohesin complex deputed to loading the complex onto double-stranded DNA. However, previous studies suggested a specific activity of NIPBL in gene transcription regulation and NIPBL binding sites, which do not overlap with those of cohesins, were identified in vitro. ${ }^{35} \mathrm{We}$, therefore, decided to analyze the effects of NIPBL downregulation on myeloid differentiation. The zebrafish represents an ideal model for studying the function of cohesin genes as knockdown can be achieved by the injection of specific oligonucleotide antisense morpholino. ${ }^{30}$ Investigating the effects of nipblb-loss-of-function during zebrafish definitive hematopoiesis, we observed an increased number of myeloid progenitors. Similar results have been obtained in different models of cohesin loss-of-function. For example, in murine models the use of short hairpin RNA against Stag2 and Smc3 generated a maturation block, delayed differentiation, and enhanced renewal of HSC, similar to the events occurring in myeloid neoplasms. ${ }^{36}$ In another study, RNA interference mouse models were created with inducible knockdown of Rad21, Smc1a and Stag2, leading to a shift in the hematopoietic stem compartment, an increased replating capacity and, over time, the development of clinical features of myeloproliferative neoplasms. ${ }^{18}$ Unlike STAG2, SMC3, SMC1A, and RAD21, NIPBL is not a recurrently mutated gene in $\mathrm{AML}{ }^{28}$ In 


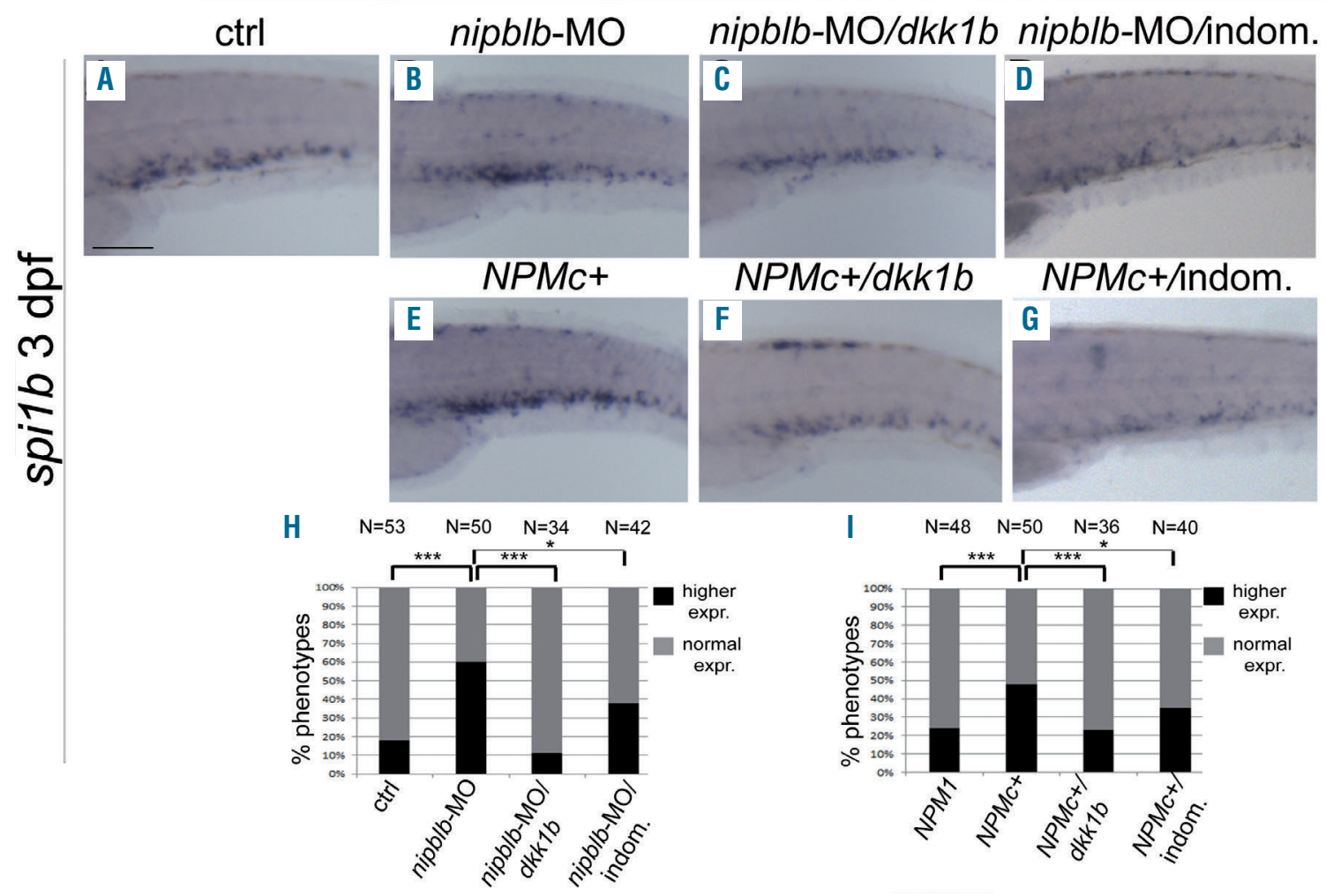

Figure 5. The impairment of myeloid cell differentiation in nipblb-MO and NPMc+ injected embryos is caused by hyper-activation of canonical Wnt signaling. (AG) Whole mount in situ hybridization analyses of spi1b. The expression of spi1b was increased in nipblb-MO (B) and NPMc+-injected embryos (E) in comparison to that in controls (A). The myeloid phenotype was rescued by injection of Wnt inhibitor dkk1b mRNA (C, F) and treatment with indomethacin (D, G). Quantification of the observed phenotypes in $(\mathrm{H})$ and $(\mathrm{I})$. Images were processed as described in the Online Supplementary Methods. The scale bar represents $100 \mu \mathrm{m}$. ${ }^{*}<0.05$ and $* * * P<0.001$. ctrl: control; MO: morpholino.

humans, heterozygous loss-of-function mutations of NIPBL cause CdLS. ${ }^{37}$ Nevertheless, CdLS patients with mutations in NIPBL or in other cohesin genes are not predisposed to myeloid neoplasms. Rare cases of leukemia have been reported in patients with CdLS, but were probably caused by other inherent aspects of this latter pathology. ${ }^{38-40}$ The mutations in cohesin genes responsible for CdLS and leukemia insurgence are considered different for their pathophysiological output: cohesin mutations in tumors occur in somatic adult cells while germline cohesin mutations in CdLS patients occur in embryonic tissue. Moreover, cohesin mutations in cancer might not trigger, but contribute to tumorigenesis only with other mutations such as TET2, ${ }^{41}$ NPM1, 9,42 DNMT3A ${ }^{43}$ or FLT3-ITD. ${ }^{44}$ Indeed, we speculate that the decrease in NIPBL expression observed in AML patients might not be due to specific somatic mutations occurring at the gene level, but rather to a secondary effect caused by genetic lesions that have arisen elsewhere (i.e., NPM1).

In nipblb-loss-of-function embryos we observed defects in myeloid differentiation with an increased number of myeloid progenitors and a decrease of mature myeloid cells. These hematopoietic defects share similarities with the myeloproliferative phenotype of NPMc+ embryos. ${ }^{12,13}$ Indeed, we also confirmed that the forced expression of $N P M c+$ generated a phenotype with expansion of HSC and myeloid precursors. However, the main difference in the hematopoietic phenotype generated by nipblb knockdown and NPMc+ forced expression was in the HSC population. In this regard, while HSC numbers were signifi- cantly increased in NPMc+ embryos, in nipblb-MO injected embryos they were almost comparable to those in controls. This finding is not surprising as we demonstrated that nipblb acts downstream of, and is regulated by, $N P M c+$. It is therefore conceivable that, also in the hematopoietic cascade that drives myeloid differentiation, the effects of nipblb are exerted on a population derived from HSC, such as myeloid progenitors.

We further observed that in nipblb knockdown and $N P M c+$ overexpressed zebrafish embryos, the Wnt/ $\beta$ catenin pathway was hyper-activated. In the process of hematopoiesis, many signaling pathways are critical during the different developmental stages of HSC and for the maturation of these cells into differentiated lineages. In particular, the canonical Wnt pathway exerts its action on specific cell populations, being fine-tuned in a dosagedependent fashion. ${ }^{45}$ We demonstrated that Nipbl plays an active role in this modulation. Indeed, in a previous study, we showed that the downregulation of nipblb in zebrafish embryos at $24 \mathrm{hpf}$ led to decreased activation of the canonical Wnt pathway. ${ }^{30}$ In this work we demonstrated that, from 48 hpf, Nipbl acts in an opposite way by hyperactivating the Wnt pathway in the whole embryo. It is conceivable that, given its double effects on the Wnt pathway, Nipblb might be considered a new factor in canonical Wnt pathway modulation.

Recently several Wnt inhibitors have been identified as potential drugs to reduce tumor cell viability in lymphoma and myeloma cell lines in vitro and in vivo. ${ }^{46}$ Thus, Wnt/ $\beta$ catenin inhibitors, such as indomethacin that has already 

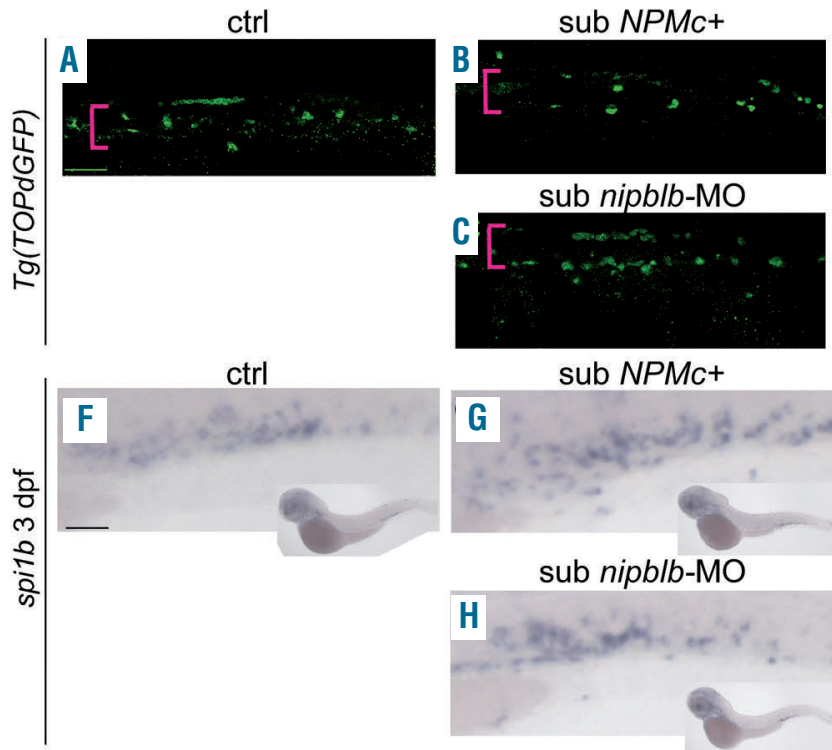

sub nipblb-MO
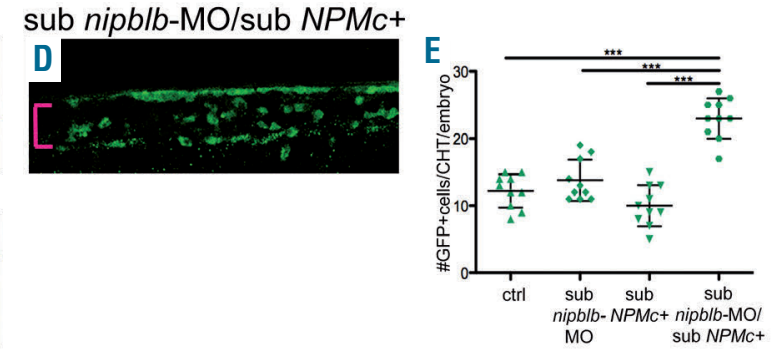

sub nipblb-MO/sub NPMc+
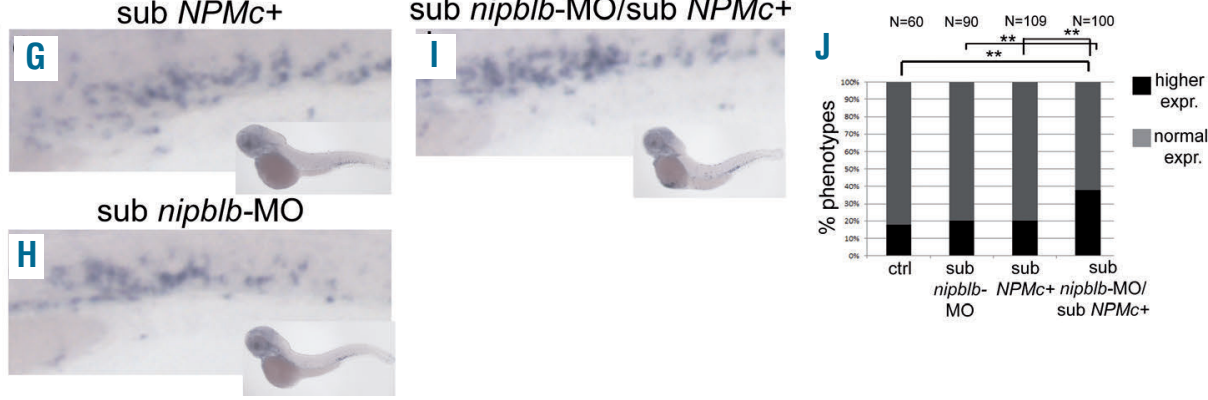

Figure 6. Co-injection of subcritical doses of nipblb-MO and NPMc+, which singularly do not have effects, indicates cooperation between NPMc+ and nipblb in myeloid differentiation. (A-D) The number of green fluorescent protein (GFP) $)^{+}$cells in caudal hematopoietic tissue (CHT) was increased when embryos at 3 dpf were co-injected with subcritical doses of both nipblb-MO and NPMc+ mRNA (D) in comparison to a single injection of a subcritical dose of NPMc+ mRNA (B) or nipblb$\mathrm{MO}(\mathrm{C})$. The numbers of GFP+ ${ }^{+}$cells in the CHT following the single injections were comparable to those in the controls (A). (E) Quantification of GFP+ cells in the CHT. (F-I) Whole mount in situ hybridization analyses of the myeloid precursor marker spilb. spilb expression was increased in embryos co-injected with subcritical doses of both nipblb-MO and NPMc+ mRNA (I) in comparison to the expression in controls and embryos injected with a subcritical dose of either NPMc+ (sub NPMc+) (G) or nipblb-MO (sub nipblb-MO) (H). (J) Quantification of the embryos presenting increased spi1b expression. Images were processed as described in the Online Supplementary Methods. The scale bar represents $100 \mu \mathrm{m}$. ${ }^{*} P<0.01$ and $* * * P<0.001$. ctrl: control; MO: morpholino.

been investigated in clinical trials ${ }^{26}$ and was able to rescue the hematopoietic phenotype in our study, could be attractive candidates for the development of new treatments for NPMc+ AML patients.

Further analyses are necessary to investigate the mechanisms through which NPMc+ interacts with NIPBL and how they regulate the canonical Wnt pathway and the hematopoietic phenotype. One hypothesis raised by previous research and our analyses is the involvement of other members of the cohesin complex such as Rad21, which is negatively regulated by Nipbl in both murine fibroblasts $^{47}$ and zebrafish (see Figure 1F). Alternatively, the aberrant cytoplasmic dislocation of mutant NPM1 might alter the normal regulation of NIPBL expression in the nucleus, similarly to what happens in the important PU.1-NPM1-mediated regulation in myeloid precursors. ${ }^{48}$ Large scale transcriptomic and/or proteomic analyses will be useful to unravel the mechanism and, to this purpose, the use of the zebrafish platform with cohesin knockdown might be a suitable model system.
In conclusion, our study correlates NIPBL with NPM1 mutations in adult AML patients for the first time and demonstrates their interplay in myeloid cell differentiation in zebrafish through involvement of the canonical Wnt pathway. The results obtained will foster the identification of new potential targets for the treatment of subgroups of AML.

\section{Acknowledgments}

We thank $R$ Monteiro, (University of Birmingham), for the Tg(CD41:GFP/kdrl:dsRED) zebrafish line, N. Bolli, (University of Milan) for useful discussion of the data, the cytometry desk staff (IFOM, Milan) for technical help in FACS experiments, $M C$ Crosti of the INGM FACS facility for sorting experiments and $M$ Spreafico and $M$ Cafora (University of Milan) for their priceless support in experimental procedures. This work was supported by the Associazione Italiana per la Ricerca sul Cancro (AIRC) (MFAG\#18714). The funders had no role in the study design, data collection and interpretation, or the decision to submit the work for publication.

\section{References}

1. Löwenberg B, Downing JR, Burnett A. Acute myeloid leukemia. N Engl J Med. 1999;341(14):1051-1062.

2. Ley TJ, Mardis ER, Ding L, et al. DNA sequencing of a cytogenetically normal acute myeloid leukaemia genome. Nature. 2008;456(7218):66-72.

3. Yan X-J, Xu J, Gu Z-H, et al. Exome sequencing identifies somatic mutations of DNA methyltransferase gene DNMT3A in acute monocytic leukemia. Nat Genet. 2011;43(4):309-315.

4. Corces-Zimmerman MR, Majeti R. Preleukemic evolution of hematopoietic stem cells: the importance of early mutations in leukemogenesis. Leukemia. 2014;28(12): 2276-2282.

5. Cancer Genome Atlas Research Network, Ley TJ, Miller C, Ding L, et al. Genomic and epigenomic landscapes of adult de novo acute myeloid leukemia. N Engl J Med. 2013;368(22):2059-2074.
6. Borer RA, Lehner CF, Eppenberger HM, Nigg EA. Major nucleolar proteins shuttle between nucleus and cytoplasm. Cell. 1989;56(3):379-390.

7. Dumbar TS, Gentry GA, Olson MOJ. Interaction of nucleolar phosphoprotein B23 with nucleic acids. Biochemistry. 1989;28 (24):9495-9501.

8. Cheng K, Grisendi S, Clohessy JG, et al. The leukemia-associated cytoplasmic nucleophosmin mutant is an oncogene with paradoxical functions: Arf inactivation and induction of cellular senescence. Oncogene. 


$$
111
$$

